University of Nebraska - Lincoln

DigitalCommons@University of Nebraska - Lincoln

January 1996

\title{
The calculation of radial dose from heavy ions: predictions of biological action cross sections
}

\author{
Robert Katz \\ University of Nebraska-Lincoln, rkatz2@unl.edu \\ Francis A. Cucinotta \\ NASA Langley Res. Center, Hampton, VA 23681, USA, francis.cucinotta@unlv.edu \\ C. X. Zhang \\ Zhongshan Univ., Guangzhou 510275, China
}

Follow this and additional works at: https://digitalcommons.unl.edu/physicskatz

Part of the Physics Commons

Katz, Robert; Cucinotta, Francis A.; and Zhang, C. X., "The calculation of radial dose from heavy ions: predictions of biological action cross sections" (1996). Robert Katz Publications. 19.

https://digitalcommons.unl.edu/physicskatz/19

This Article is brought to you for free and open access by the Research Papers in Physics and Astronomy at DigitalCommons@University of Nebraska - Lincoln. It has been accepted for inclusion in Robert Katz Publications by an authorized administrator of DigitalCommons@University of Nebraska - Lincoln. 


\title{
The calculation of radial dose from heavy ions: predictions of biological action cross sections
}

\author{
Robert Katz ${ }^{a, *}$, Francis A. Cucinotta ${ }^{b}$, C.X. Zhang ${ }^{c}$ \\ ${ }^{a}$ University of Nebraska, Lincoln, NE 68588-0111, USA \\ ${ }^{\mathrm{b}}$ NASA Langley Res. Center, Hampton, VA 23681, USA \\ 'Dept. of Physics, Zhongshan Univ., Guangzhou 510275, China
}

\begin{abstract}
The track structure model of heavy ion cross sections was developed by Katz and co-workers in the 1960s. In this model the action cross section is evaluated by mapping the dose-response of a detector to $\gamma$ rays (modeled from biological target theory) onto the radial dose distribution from $\delta$ rays about the path of the ion. This is taken to yield the radial distribution of probability for a "hit" (an interaction leading to an observable end-point). Radial integration of the probability yields the cross section. When different response from ions of different $Z$ having the same stopping power is observed this model may be indicated. Since the 1960s there have been several developments in the computation of the radial dose distribution, in the measurement of these distributions, and in new radiobiological data against which to test the model. The earliest model, by Butts and Katz, made use of simplified $\delta$ ray distribution functions, of simplified electron range-energy relations, and neglected angular distributions. Nevertheless it made possible the calculation of cross sections for the inactivation of enzymes and viruses, and allowed extension to tracks in nuclear emulsions and other detectors and to biological cells. It set the pattern for models of observable effects in the matter through which the ion passed. Here we outline subsequent calculations of radial dose which make use of improved knowledge of the electron emission spectrum, the electron range-energy relation, the angular distribution, and some considerations of molecular excitation, of particular interest both close to the path of the ion and the outer limits of electron penetration. These are applied to the modeling of action cross sections for the inactivation of several strains of $E$-coli and $B$. subtilis spores where extensive measurements in the "thin-down" region have been made with heavy ion beams. Such calculations serve to test the radial dose calculations at the outer limit of electron penetration. We lack data from which to test these calculations in regions close to the path of the ion aside from our earliest work on latent tracks in plastics, though it appears that the criterion then suggested for the threshold of track formation, of a minimal dose at a minimal distance (of about $20 \AA$, in plastics), remains valid.
\end{abstract}

\section{Model of radial dose from heavy ions}

In the passage of radiation through matter there are several contributions to the stopping power or linear energy transfer (LET), including excitation of the medium, ionization of atomic electrons and nuclear stopping. For many problems the track structure, represented by the radial distribution of dose deposited by $\delta$ rays is determining, with excitation and nuclear stopping playing a negligible role. The energy density distribution (radial dose) as a function of radial distance $t$ from the path of the ion was first formulated by Butts and Katz [1], and subsequently by Kobetich and Katz [2], Zhang et al. [3] and many others. The model of Kobetich and Katz has recently been updated [4] to include a better model of the angular distribution, newer data on range-energy relations by Tabata et al.

\footnotetext{
* Corresponding author.
}

[5], an empirically based model of $\delta$ ray production by Rudd [6], molecular excitations based on Monte Carlo calculations in liquid water by Waligorski et al. [7]. Results of these calculations, with a variety of assumptions about angular dependence and molecular excitations are given by Cucinotta et al. [4]. Even this has been reconsidered through subsequent use of the formulation of excitations by Brandt and Ritchie [8], who point out that excitations from distant collisions are confined to the infratrack radius, defined by the Bohr adiabatic criterion. The result of our calculations, for $1 \mathrm{MeV}$ protons in water using different assumptions (to display problems encountered close to and remote from the path of the ions) and ${ }^{20} \mathrm{Ne}$ at $377 \mathrm{MeV} / \mathrm{amu}$, and in which the excitation functions of Brandt and Ritchie are incorporated, are shown in Figs. 1a and $1 \mathrm{~b}$, in comparison with measurements by Wingate and Baum [9] for protons and measurements of Varma and Baum [10] for Ne. The present calculations made for other ions (adjusted from calculations for protons by multiplica- 

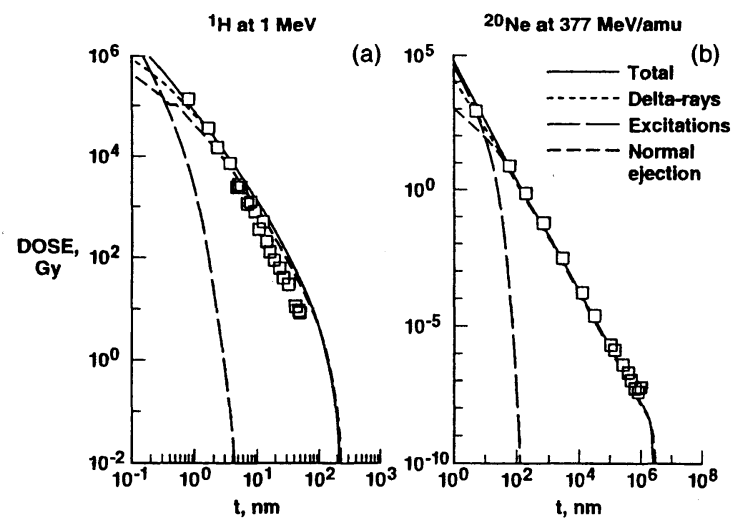

Fig. 1. (a) Radial dose distribution from $1 \mathrm{MeV}$ protons in water, calculated under different assumptions, as compared with experimental data obtained in gases. (b) Radial dose distribution in water from ${ }^{20} \mathrm{Ne}$ at $377 \mathrm{MeV} / \mathrm{amu}$ compared with data obtained in gases.

tion with the square of the effective charge) are here used for the evaluation of inactivation cross sections for bacteria and bacterial spores, and for the evaluation of cross sections for HGPRT mutations. Typically different assumptions yield major differences close to the path of the ion (most important for latent tracks and possibly for consideration of damage to crystalline structure) and remote from the path of the ion (most important for considerations of "thin down", the decrease in the inactivation cross section while the LET of the ion increases, as the ion approaches the end of its range). Radial dose calculations for protons of different energies $(1,10,100 \mathrm{MeV} / \mathrm{amu})$ in carbon, silicon, and gold are shown in Fig. 2. These calculations are based on a variety of input data obtained from measurements in gases, from measurements in solids, as well as from theoretical considerations.

The model for the radial dose from $\delta$ rays described above can be parameterized by utilizing the $1 / t^{2}$ fall off dependence at intermediate distances and introducing functions that modify the distribution at small and large distances. The radial dose in water is then

$D(t)=\left(Z^{* 2} / \beta^{2}\right)\left(N e^{4} / m c^{2}\right) f_{\mathrm{S}}(t)\left(1 / t^{2}\right) f_{\mathrm{L}}(t)$,

where $\beta c$ is the velocity of the ion, $Z^{*}$ is the effective charge and $m$ the electron mass. The function $f_{\mathrm{S}}(t)$ modifies the short distance behavior and is represented by

$f_{\mathrm{S}}(t)=\left(1 / t+c_{1}\right)^{-1}$,

with

$c_{1}=0.6+1.7 \beta$.

The function $f_{\mathrm{L}}(t)$ modifies the long distance behavior and is represented by

$f_{\mathrm{L}}(t)=\exp -\left(t / 0.37 T_{\max }\right)^{2}$,

where $T_{\max }$ is the maximum radial penetration distance for $\delta$ rays of an ion at speed $\beta c$.

Comparisons of the results of this parameterization with calculations they represent are shown in Fig. 3.

\section{The track structure model}

The track structure model is based on the assumption that the effects produced by secondary electrons from $\gamma$ rays and from the $\delta$ rays from heavy ions are comparable

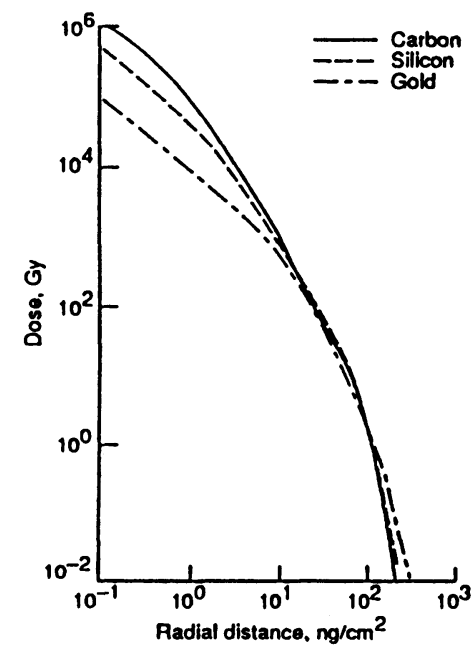

$1 \mathrm{MeV}$

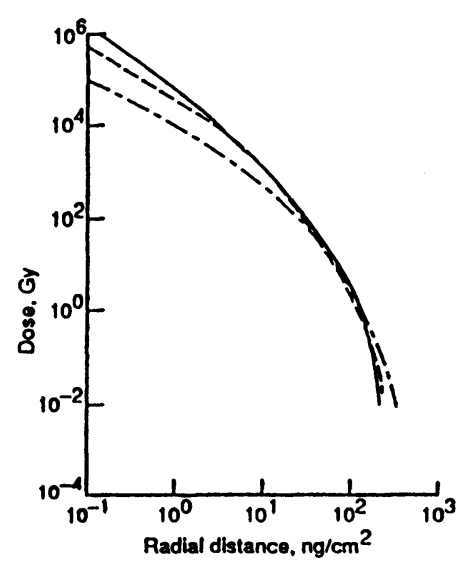

$10 \mathrm{MeV}$

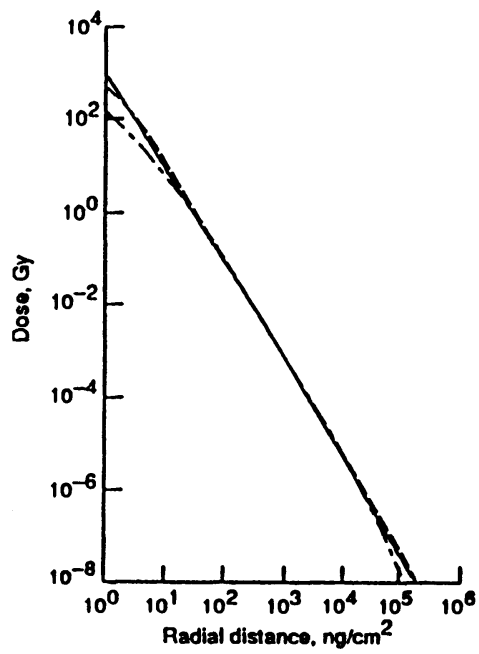

$100 \mathrm{MeV}$

Fig. 2. Radial dose from protons at 1,10 , and $100 \mathrm{MeV}$ in carbon, silicon and gold, calculated from the present algorithm. 


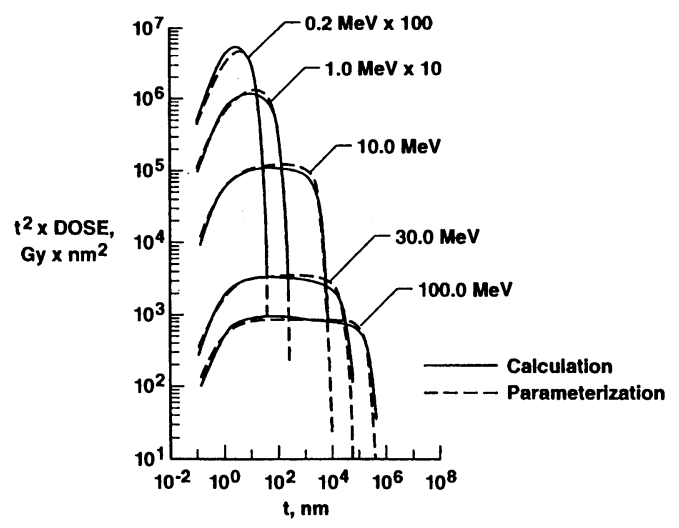

Fig. 3. Calculations of the radial dose from the parameterization of Eqs. (1)-(4) compared to the calculations on which they are based.

at the same dose. The time scale of electron depositions and differences in the initial electron energy spectrum are thus not considered in the model. The neglect of differences in the initial electron energy spectrum are made plausible if we assume that the electron slowing down spectrum [11] is responsible for the radiation action. The effects of including primary excitations in the radial dose model in relation to the response of the detector are little understood, and are here neglected.

For such effects as latent tracks in etchable materials we assumed (in 1968) a threshold condition based on minimal dose (required to produce etchable damage in bulk matter) at a minimal radius taken to be about $20 \AA$ in plastics [12]. This criterion is supported by recent investigations [13], with the minimal radius taken to be about 35 $\AA$ in an amorphous metallic material [14].

For many other effects the response to $\gamma$ rays is represented through biological target theory, a statistical model in which we speak of the probability for making a hit in a detector through a multi-hit or a multi-target formula [15]. We speak of a characteristic dose, $D_{0}$, at which there is an average of 1 hit per target found from the response to $\gamma$ rays, and take the average number of hits per target at radial distance $t$ to be the dose at distance $t$, $D(t)$, divided by $D_{0}$. In formulating the effects produced by heavy ions it is useful to imagine the energy deposited in coaxial cylindrical shells surrounding many ions. The radial dose in a shell is an average quantity over a large volume made up of equivalent shells about many ions. The probability for producing effects in a macroscopic volume by a given dose of $\gamma$ rays is used to estimate the probability for producing effects in a shell about a single ion. In this way one can represent the tracks of heavy ions in nuclear emulsions [15]. When the action cross section is the measured quantity, it is calculated by summing the probability for causing an effect over all of the shells, the radial integral of the probability over all the shells is the theoretical value of the action cross section. This calculation parallels the classical calculation of the Rutherford scattering cross section. There the effect is moderated by the long range Coulomb interaction. In the present calculation the long range interaction arises from the energy deposited by $\delta$ rays. For an $m$ (ulti) target interaction where $m$ is the number of targets which must be hit to cause an observable effect we find the cross section $\sigma$ as

$\sigma=2 \pi \int t \mathrm{~d} t\left[1-\mathrm{e}^{-\left(D(t) / D_{0}\right)}\right]^{m}$.

For an extended target we average the radial dose distribution over the target volume assumed to be a short cylinder of radius $a_{0}$ whose axis is parallel to the path of the ion. For one hit detectors $m=1$, a single hit in a single target suffices to inactivate the detector. For biological cells $m$ is frequently 2 or more, and the complex character of the structure of the nucleus of a cell in which the targets for inactivation and mutation are contained requires a more elaborate model [16]. For the present discussion we direct our attention to the inactivation of spores of Bacillus subtilis [17], E. Coli $B_{\mathrm{s}-1}$ and $B / r$ [18] for which extensive data exist giving the response to $\gamma$ rays and to a series of heavy ion bombardments which we use as a test of our radial dose calculations, particularly at large distances from the path of the ion, and as a further test of our overall model of the cross section for 1-hit detectors. We also calculate the cross section for observable HGPRT mutations in V79 cells with a more complex model.

\section{Inactivation cross section: Bacillus subtilis spores (rec-), $E$-Coli $B / r$ and $B_{s-1}$}

Calculations for the action cross sections of 1-hit detectors irradiated with heavy ions of different energies require knowledge of the radial dose distribution, the radius $a_{0}$ of the target, and the characteristic dose $D_{0}$, often called $(D-37)$, at which $37 \%$ of the targets survive an irradiation with $\gamma$ rays. Identification as a 1-hit detector arises from an exponential response to dose of $\gamma$ rays, displayed by these three cell systems. For 1-hit detectors the cross section is given as

$\sigma=L /\left(D_{\mathrm{i}}-37\right)$,

where $L$ is the LET of the bombarding ion and $\left(D_{\mathrm{i}}-37\right)$ is the dose of ions at which there is $37 \%$ survival. This result arises from equating $\sigma\left(F_{\mathrm{i}}-37\right)$ to $D /\left(D_{\mathrm{i}^{-}}-37\right)$, where $\left(F_{\mathrm{i}^{-}}\right.$ 37 ) is the particle fluence for $37 \%$ survival, and $F L=D$.

Calculations for the inactivation cross sections of $B$. Subtilus spores, $E$-coli $B / r$ and $B_{s-1}$ were made by Zhang and Katz [19] utilizing a radial dose distribution calculated by Zhang et al. [3] which used other assumptions about the $\delta$ ray distribution formula, a classical angular distribution, and a different range-energy relation 
(a)
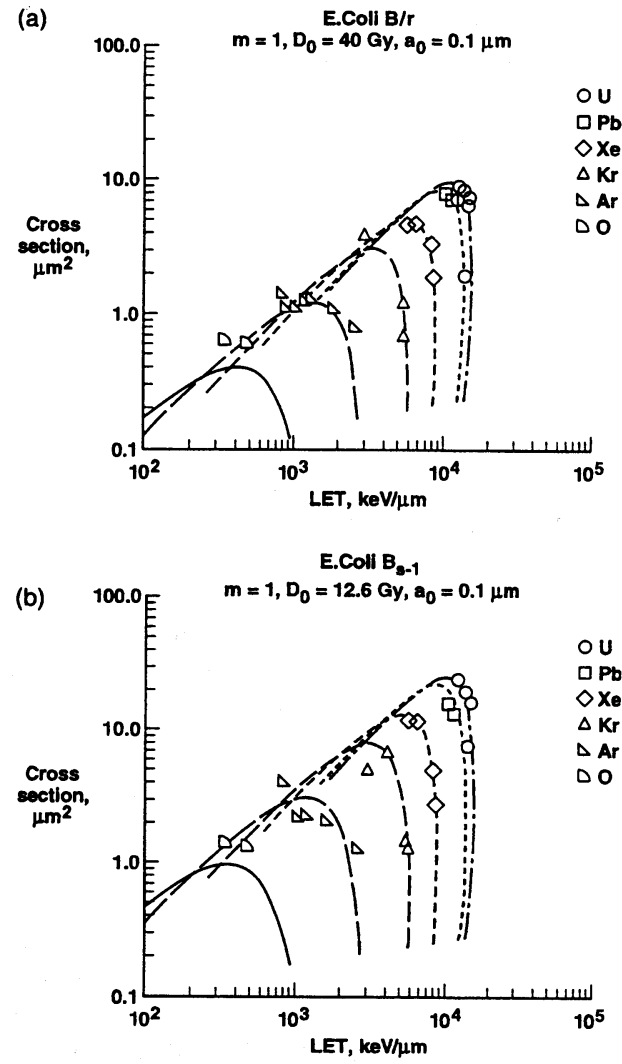

Fig. 4. (a) Calculated inactivation cross sections for $E$. Coli $B / r$, with data superimposed. (b) Calculated inactivation cross sections for $E$. Coli $B_{\mathrm{s}-1}$ with data superimposed.

for electrons. Here we seek to examine the results of an improved radial dose distribution which makes use of the most recent available data and to compare the findings with the earlier work.

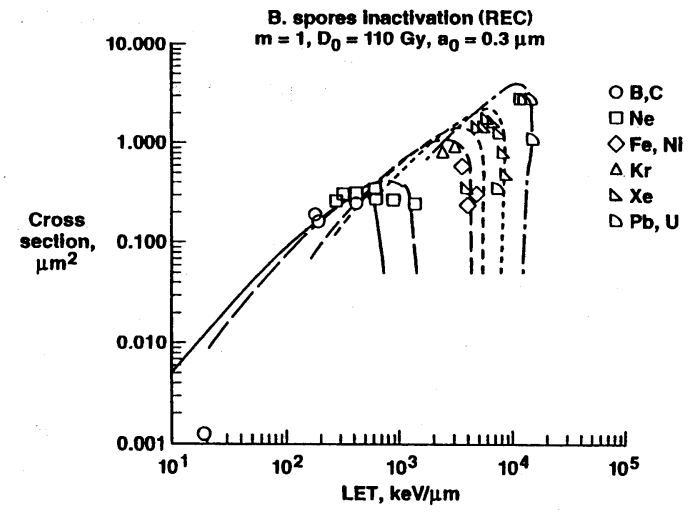

Fig. 5. Calculated inactivation cross sections for Bacillus subtilis spores with data superimposed.

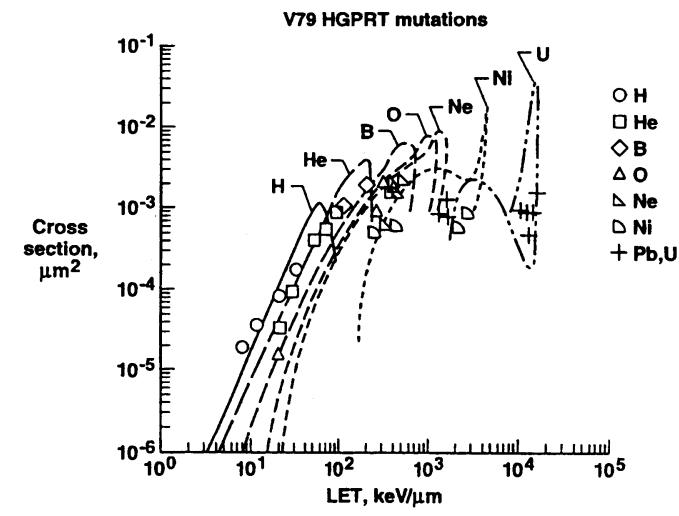

Fig. 6. Calculated cross sections for V79 HGPRT mutations vs. LET with data superimposed.

Values of $D_{0}$, the $D-37$ for $\gamma$ rays, for these cells differ among different investigators. Thus for $B / r$ Takahashi et al. [20] find 36.5 Gy while Schäfer et al. find 47.6 Gy. We have chosen a value of $40 \mathrm{~Gy}$ for best fit of our calculations to the heavy ion data [18]. For $B_{s-1}$ Takahashi reports $12.6 \mathrm{~Gy}$ while Schäfer reports $15.4 \mathrm{~Gy}$. Here we have chosen $12.6 \mathrm{~Gy}$, for best fit of calculations to data. Calculated cross sections for a variety of heavy ion bombardments are shown in Figs. $4 \mathrm{a}$ and $4 \mathrm{~b}$, with data points superimposed.

For the inactivation of Bacillus subtilis spores (rec-) we have chosen $D_{0}=110 \mathrm{~Gy}$, as compared to the experimental value of $93 \pm 7.6$ Gy [17]. Calculated cross sections are shown in Fig. 5, with data points superimposed.

Our results do not materially differ from those of Zhang and Katz, though the parameters for best fit differ somewhat. The values of $D_{0}$ chosen by Zhang and Katz for $E$. Coli $B / R, B_{s-1}$ and spores are $44.6 \mathrm{~Gy}, 13.8 \mathrm{~Gy}$ and $105 \mathrm{~Gy}$, while the values chosen in the present work are $40 \mathrm{~Gy}, 12.6 \mathrm{~Gy}$ and $110 \mathrm{~Gy}$. In all cases acceptable fits of calculations to data are achieved.

\section{Mutation induction}

Individual tracks of heavy ions deposit large energy in a cell nucleus with inactivation cross sections approaching or exceeding the area of a cell nucleus. To account for inactivation effects on recovery of mutants, correlation of lesion sites for inactivation and gene mutation is considered relative to the radial dose distribution. The action cross sections for the probability to recover a mutant from a single ion track considers the dose at two sites, one for mutation, and the other for inactivation randomly located in the nuclear volume. The results of such a calculation are shown in Fig. 6, compared to data for HGPRT mutations in V79 cells [22,23]. A more detailed description of this calculation will be published elsewhere. 


\section{Discussion}

While we prefer the present radial dose calculations on a priori basis it is clear that the dominant contribution to the successful result is the model itself, and that we will need more precise data to make a proper test of the radial dose distribution. Hopefully such measurements will be made with dry enzymes and viruses where we have already achieved excellent agreement between data and calculations at bombardments where thin down was not achieved experimentally [21].

\section{Acknowledgements}

This work is supported by the United States Department of Energy, The National Aeronautics and Space Administration and the National Natural Science Foundation of China.

\section{References}

[1] J.J. Butts and R. Katz, Radiat. Res. 30 (1967) 855.

[2] E.J. Kobetich and R. Katz, Phys. Rev. 170 (1968) 391.

[3] C.X. Zhang, X.W. Liu, M.F. Li and D.L. Luo, Radiat. Prot. Dosim. 52 (1994) 93.

[4] F.A. Cucinotta, R. Katz, J.W. Wilson and R.R. Dubey, NASA Technical Paper 3497 (1995), NASA Langley Research Center, Hampton, VA 23681-0001, USA.

[5] T. Tabata, R. Ito and S. Okabe, Nucl. Instr. and Meth. 103 (1972) 85.
[6] M.E. Rudd, Nucl. Tracks Radiat. Meas. 16 (1989) 213.

[7] M.P.R. Waligorski, R.N. Hamm and R. Katz, Nucl. Tracks. Radiat. Meas. 11 (1986) 309.

[8] W. Brandt and R.H. Ritchie, in Physical Mechanisms in Radiation Biology, eds. R.D. Cooper and R.W. Wood, Technical Information Services, US Atomic Energy Commission (1954) p. 20.

[9] C.L. Wingate and J.W. Baum, Radiat. Res. 65 (1976) 1.

[10] M.N. Varma and J.W. Baum, Radiat. Res. 81 (1980) 355.

[11] R.N. Hamm, H.A. Wright, R. Katz, J.E. Turner and R.H. Ritchie, Phys. Med. Biol. 23 (1978) 1149.

[12] R. Katz and E.J. Kobetich, Phys. Rev. 170 (1968) 401.

[13] O.A. Bernoala, G. Saint-Martin and C. Grasso, Nucl. Tracks Radiat. Meas. 22 (1993) 25.

[14] C. Trautmann, M. Toulemonde, C. Dufour and E. Paumier, GSI (Darmstadt) Report 94-1 (1994) 201.

[15] R. Katz, Nucl. Track Detection 2 (1978) 1.

[16] R. Katz, R. Zachariah, F.A. Cucinotta and C.X. Zhang, Radiat. Res. 140 (1994) 356.

[17] K. Baltschukat and G. Horneck, Radiat. Environ. Biophys. 30 (1991) 87.

[18] M. Schäfer, C. Schmitz and R. Bücker, Radiat. Prot. Dosim. 52 (1994) 233.

[19] C.X. Zhang and R. Katz, Chin. Sci. Bull. (in press).

[20] T. Takahashi, F. Yatagai, S. Konno, T. Katayama and I. Kaneco, Adv. Space Res. 6 (1986) 117.

[21] M.P.R. Waligorski, K.S. Loh and R. Katz, Radiat. Phys. Chem. 30 (1987) 201.

[22] T. Kranert, E. Schneider and J. Kiefer, Int. J. Radiat. Biol. 58 (1990) 979.

[23] M. Belli, F. Cera, R. Cherubini, A.M.I. Haque, F. Ianzini, G. Moschini, O. Sapora, G. Simone, M.A. Tabocchini and P. Tiverton, Int. J. Radiat. Biol. 63 (1993) 331. 